in invasive infections. The decreased expression of lectin pathway proteins in neonates may contribute to their extraordinary vulnerability for bacterial infections. Low MBL concentrations appear to be an important susceptibility factor for gram-negative sepsis, and low $\mathrm{H}$-ficolin for gram-positive sepsis. In contrast, M-ficolin reflects phagocytic activity and was elevated in early-onset sepsis.

79

\section{MANNOSE BINDING LECTIN (MBL) YY GENOTYPE IS ASSOCIATED WITH AN INCREASED RISK OF NECROTIZING ENTEROCOLITIS(NEC) IN PRETERM NEONATES IN NICU}

C. Auriti ${ }^{1}$, G. Prencipe ${ }^{1}$, C. Massai ${ }^{2}$, R. Devito $^{3}$, R. Inglese ${ }^{4}$, M.P. Ronchetti ${ }^{1}$, F. Piersigilli ${ }^{5}$, F. Morini ${ }^{1}$, G. Seganti ${ }^{1}$, C. Azzari ${ }^{2}$, F. De Benedetti ${ }^{6}$

${ }^{1}$ Medical and Surgical Neonatology, Bambino Gesù Children's Hospital, Rome, ${ }^{2}$ Department of Pediatrics, Meyer Institute, Florence, ${ }^{3}$ Department of Histopathology, Bambino Gesù Children's Hospital Rome, ${ }^{4}$ Laboratory of Clinical Chemistry, Bambino Gesù Children's Hospital, ${ }^{5}$ Medical and Surgical Neonatology, Bambino Gesù Children's Hospital Rome, ${ }^{6}$ Laboratory of Rheumatology, Bambino Gesù Children's Hospital, Rome, Italy

Background: MBL initiates complement activation and could have a role in the pathogenesis of ischemia-reperfusion pathologies, such as NEC. We investigated the association between MBL2 genotype, the development of NEC and the MBL expression on specimens of bowel tissue in a cohort of preterm neonates in NICU.

Material and methods: We determined the MBL2 genotype in 118 preterm neonates admitted in NICU. The exon 1 SNPs 52,54, 57 and SNPs -550 and -221 of the promoter region of the MBL2 gene were also determined. Immunoistochemical analysis was performed on 16 bowel bioptic paraffin-embedded specimens, collected during surgery in neonates with NEC.Clinical cases and summary results:21/118 neonates developed NEC. The analysis of the polymorphisisms in the promoter region at-221(variant $X / Y$ ) showed that the frequency of the $Y Y$ genotype was $85.7 \%$ in the neonates with NEC and $53.6 \%$ in the controls $(p=0.013)$. Adjusting for gestational age, the association between YY genotype and the development of NEC was still significant $(O R=5.02 ; p=0.015)$. $M B L$ was highly expressed in anastomotic healthy tissue, in enterocytes and istiocytes. No MBL staining was detectable in the necrotic bowel tissue. Neonates MBL low and deficient producer showed negligible or weak staining for MBL.Conclusions: YY genotype of the promoter of the MBL2 gene appeared to increase the risk of developing NEC. The epithelial localization of MBL in the bowel is consistent with a role for $\mathrm{MBL}$ in developing full-thickness necrosis during NEC.

\section{0}

\section{MICROARRAY INVESTIGATION OF HOST RNA EXPRESSION PROFILES IN NEONATAL INFECTION}

C.L. Smith ${ }^{1,2}$, P. Dickinson ${ }^{2}$, M. Craigon ${ }^{2}$, A. Ross ${ }^{2}$, M.R. Khondoker ${ }^{2}$, T. Forster ${ }^{2}$, A. Ivens ${ }^{3}$, A. Jackson'1, P. Lacaze ${ }^{2}$, B.J. Stenson ${ }^{1}$, P. Ghazal ${ }^{2}$

${ }^{1}$ Neonatal Unit, Simpson Centre for Reproductive Health, ${ }^{2}$ Division of Pathway Medicine, ${ }^{3} \mathrm{FIOS}$ Genomics, University of Edinburgh, Edinburgh, UK

Background and aims: Infection causes significant neonatal morbidity and mortality. Currently available methods for diagnosing infection are unreliable. We aimed to examine differences in host RNA expression profiles between infants with confirmed infection and control infants using microarray technology.

Methods: RNA was extracted from neonatal whole blood taken from infants with confirmed infection and from controls using a modified PAXgene ${ }^{\mathrm{TM}}$ Blood RNA system protocol. High quality RNA was run on Illumina ${ }^{\circledR}$ Human Whole-Genome Expression BeadChip microarrays. Normalised, validated microarray data was analysed to examine differences between control and infected samples. Functional annotation according to gene ontology and pathway analysis was performed.

Results: 28 infected and 35 control samples were examined. Differential gene expression between infected and control groups was analysed: 448 features had $>2$-fold up-regulation and 341 features $>2$-fold down-regulation ( $p<0.001$ ) in infected compared to control infants. There was significant immune-related differential gene expression. Upregulated genes in the infected group included genes involved in cytokine, complement, interferon and Toll Like Receptor related processes. Downregulated genes included genes involved in antigen processing, MHC II activity and T cell activation and signalling. 
Conclusions: There is immune-related differential gene expression between infected and control infants. Many of our results corroborate findings previously published for adult and paediatric populations. In addition, these results provide evidence that neonates are capable of mounting a substantial immune response to infection. It is likely that, with larger studies and, with examination of training sets of data, immune gene expression signatures for neonatal infection can be defined.

\section{1}

\section{METABOLOMIC ANALYSES SHOW FAILURE OF ACYLCARNITINE METABOLISM AND INCREASED OXIDATIVE STRESS IN LIVER OF GRACILE (BCS1L $\left.{ }^{\mathrm{G} / \mathrm{G}}\right)$ MICE}

\author{
H. Kotarsky ${ }^{1}$, P. Levéen ${ }^{1}$, D. Enot ${ }^{2}$, M. Keller ${ }^{3}$, \\ V. Fellman ${ }^{1,4}$
}

${ }^{1}$ Dept. Pediatrics, Lund University, Lund, Sweden,

${ }^{2}$ Biocrates Life Sciences AG, Innsbruck, Austria,

${ }^{3}$ Dept. Pediatrics, University Hospital Essen, Essen, Germany, ${ }^{4}$ Dept. Pediatrics, University of Helsinki, Helsinki, Finland

Background and aims: BCS1L is a chaperone assembling the Rieske iron-sulphur protein into respiratory chain complex III. To study BCS1L functions and pathophysiology of GRACILE syndrome (MIM 603358), we have introduced the disease mutation (Bcs1/ 232A> G) into mice, using gene targeting. The homozygous mutant mice $\left(B \operatorname{cs} 1 /^{G / G}\right)$ exhibit a lethal disease after $24 \mathrm{~d}$ of age resembling GRACILE syndrome: growth restriction, hepatopathy, tubulopathy, and progressive complex III deficiency. We aimed to assess metabolic changes in liver of $B c s 1 / / / G$ mice to characterize genotype-metabolomic phenotype correlation.

Methods: Bcs 1/G/G mice and littermate controls were sacrificed at age 7 (14 pairs) and $24 \mathrm{~d}$ (symptomless, 8 pairs), and $5 \pm 1$ wk (affected, 18 pairs). Snap-frozen liver samples were stored in $80^{\circ} \mathrm{C}$. Metabolomic analyses of 199 metabolites (amino acids, series of carnitines and lipids, HODE, HETE, bile acids, metabolites of the energy metabolism) were carried out at BIOCRATES Life Sciences AG.

Results: Profound changes in liver metabolite levels (increase in medium and long chain acylcarnitines and decrease in short chain acylcarnitines) were found in $5 \pm 1 \mathrm{wk}$, and slight changes in $24 \mathrm{~d} B c s 1 / \mathrm{G} / \mathrm{G}$ animals. Markers of oxidative stress (15S-HETE, 12S-HETE, 13-HODE and methioninsulfoxide), bile acids, amino acids, biogenic amino acids, sphingolipids and phosphatidylcholines were significantly increased in affected $B c s 1 /^{\mathrm{G} / \mathrm{G}}$ animals only. Hexose-phosphate, lactate, alpha - ketoglurate were decreased compared to littermates

Conclusions: Affected Bcs1/G/G mice with progressive complex III deficiency have major changes in acylcarnitine metabolism, which seems to precede oxidative stress and profound changes in metabolites indicating cell injury as well as liver dysfunction.

\section{2}

\section{DOES THE POSITION IN PREGNANCY INFLUENCE THE MATURATION OF HIP JOINT?}

\author{
C. Casini, R. Zambardi, C. Filippelli, M.P. Villa \\ UOC Pediatria, University 'La Sapienza,' II Faculty \\ of Medicine, Rome, Italy
}

Purpose: To evaluate the role of fetal position (left, right, cephalic, breech) in the development of hip joint, in an unselected population of babies with normal hips.

Methods: 672 outpatients, aged between 3 and 22 weeks (mean age $=9,85 \pm 2,06$ weeks; M / F $=340 / 332$ ) visited at the UOC of Pediatria in 2009 have been recruited. All studied subjects had mature hips (type la ) and were examined by ultrasounds with Graf's method .

Results: The value of alfa angle, which expresses the bony coverage, was not statistically different in right and left hip $(68,18 \pm 4,10$ vs $68,51 \pm 3,86$, $\mathrm{p}=\mathrm{ns}$ ). The value of beta angle, which expresses cartilaginous coverage, was higher in right hip $(48,14,2 \pm 4,47$ vs $47,59 \pm 4,54, p=0,002)$. The direction of the baby (cephalic or breech) during pregnancy did not influence the bony, nor the cartilaginous covering, nor the age of withdrawal of nucleus of ossification of femoral head in normal hips.

Conclusions: Our study shows that cartilaginous coverage of the femoral head is better in right hip than in left hip; this could be related to the higher frequency of occipital-iliac left anterior position in uterus, that keeps the right hip more stable, helping the cartilaginous covering .Further studies are needed to confirm these observations. 